\title{
Thermal emission, shock modification, and X-ray emitting ejecta in SN 1006
}

\author{
M. Miceli ${ }^{1,2,3}$, F. Bocchino ${ }^{2,3}$, D. Iakubovskyi ${ }^{4}$, S. Orlando ${ }^{2,3}$, I. Telezhinsky ${ }^{5}$, M. G. F. Kirsch ${ }^{6}$, O. Petruk ${ }^{3,7,8}$, \\ G. Dubner ${ }^{9}$, and G. Castelletti ${ }^{9}$ \\ 1 Dipartimento di Scienze Fisiche ed Astronomiche, Sezione di Astronomia, Università di Palermo, Piazza del Parlamento 1, \\ 90134 Palermo, Italy \\ e-mail: miceli@astropa.unipa.it \\ 2 INAF - Osservatorio Astronomico di Palermo, Piazza del Parlamento 1, 90134 Palermo, Italy \\ 3 Consorzio COMETA, via S. Sofia 64, 95123 Catania, Italy \\ ${ }^{4}$ Bogolyubov Institute for Theoretical Physics, Metrologichna str. 14-b Kiev 03680, Ukraine \\ 5 Astronomical Observatory, Kiev National Taras Shevchenko University, Kiev 04053, Ukraine \\ ${ }^{6}$ European Space Operations Centre, ESA, Robert-Bosch-Str. 5, 64293 Darmstadt, Germany \\ 7 Institute for Applied Problems in Mechanics and Mathematics, Naukova St. 3-b, Lviv 79060, Ukraine \\ 8 Astronomical Observatory, National University, Kyryla and Methodia St. 8, Lviv 79008, Ukraine \\ 9 Instituto de Astronomía y Física del Espacio (IAFE), CC 67, Suc. 28, 1428 Buenos Aires, Argentina
}

Received 11 December 2008 / Accepted 18 March 2009

\section{ABSTRACT}

\begin{abstract}
Context. Efficient particle acceleration can modify the structure of supernova remnants. We present the results of a combined analysis of the XMM-Newton EPIC archive observations of SN 1006.

Aims. We attempt to describe the spatial distribution of the physical and chemical properties of the X-ray emitting plasma at the shock front. We investigate the contribution of thermal and non-thermal emission to the X-ray spectrum at the rim of the remnant to study how the acceleration processes affect the X-ray emitting plasma.

Methods. We perform a spatially resolved spectral analysis of a set of regions covering the entire rim of the shell and we apply our results in producing a count-rate image of the "pure" thermal emission of SN 1006 in the 0.5-0.8 keV energy band (subtracting the non-thermal contribution). This image differs significantly from the total image in the same band, especially close to the bright limbs. Results. We find that thermal X-ray emission can be associated with the ejecta and study the azimuthal variation in the physical and chemical properties of the ejecta by identifying anisotropies in the temperature and chemical composition. By employing our thermal image, we trace the position of the contact discontinuity over the entire shell and compare it with that expected from 3D MHD models of SNRs with an unmodified shock.

Conclusions. We conclude that the shock is modified everywhere in the rim and that the aspect angle between the interstellar magnetic field and the line of sight is significantly lower than $90^{\circ}$.
\end{abstract}

Key words. X-rays: ISM - ISM: supernova remnants - ISM: individual objects: SN 1006

\section{Introduction}

The diffusive Fermi acceleration process in supernova remnant (SNR) shocks can account for the observed spectrum of galactic cosmic rays up to at least its knee at $3 \times 10^{15} \mathrm{eV}$ and at even higher energies (Berezhko \& Völk 2007; Blandford \& Eichler 1987). The ASCA detection of synchrotron X-ray emission in SN 1006 showed that electrons can be accelerated up to very high energy at the shock front, providing direct proof at the connection between SNRs and cosmic-ray acceleration (Koyama et al. 1995).

The loss of energy "deposited" in the production of relativistic particles and their non-linear back-reaction on the background plasma are predicted to strongly affect the structure of the remnant, by increasing the shock compression ratio well above the Rankine-Hugoniot limit and decreasing both the post-shock temperature and the geometric extension of the region between the main shock front and the contact discontinuity (Berezhko \& Ellison 1999; Decourchelle et al. 2000; Blasi 2002, and references therein). All these effects modify the spectral properties of the thermal emission. Therefore, both thermal and non-thermal emission can provide important information about the acceleration processes and can be useful in verifying the expected effects of the shock modification and studying the open questions still under debate. These questions include: (1) what are the factors influencing the fraction of particles injected in the acceleration process? (2) What is their maximum energy; and (3) what is the relationship between the upstream/downstream magnetic fields and the acceleration processes?

To this end SN 1006 is an ideal target. Its rather simple morphology, the precise knowledge of its age, and its evolution in a low-density, uniform medium (because of its high galactic latitude) makes it an ideal laboratory for these studies. The bilateral morphology of SN 1006 shows two opposed radio and X-ray bright limbs characterized by non-thermal emission separated by a region at low surface brightness. The synchrotron limbs reveal narrow filaments in both radio and X-rays, the X-ray emission exhibiting more significant surface brightness inhomogeneities (see, for example, Rothenflug et al. 2004). As pointed out by Rothenflug et al. (2004), the very low surface brightness above $2 \mathrm{keV}$ in the interior strongly suggests that the ambient magnetic field is parallel to the shock velocity in the bright limbs 
(polar-cap scenario), in the framework of the quasi-parallel model for the injection (i.e., the injection efficiency of the accelerated particles increases with decreasing obliquity angle, $\psi$, between the magnetic field and the shock velocity). Rothenflug et al. (2004) studied the azimuthal variations in the spectral properties of the non-thermal emission over the entire shell and its radial profile from the center to the north-eastern bright limb. They found that the roll-off frequency of the synchrotron emission is much higher in the bright non-thermal limbs than in the center and elsewhere in the rim. These results are difficult to explain in terms of the polar cap scenario, but are also unexpected in the "equatorial belt" scenario (i.e., in which the ambient magnetic field, $B_{0}$, is oriented from south-east to north-west and the injection efficiency is higher when $\psi \sim 90^{\circ}$ ), which predicts variations of a factor $\sim 2$, much less than the observed factor $>10$. Petruk et al. (2008) showed that, by assuming a uniform ambient magnetic field in the polar-cap scenario, SN 1006 should be a centrally filled SNR in radio data to reproduce the observed azimuthal radio profiles, which is the converse of what is observed. The determination of the magnetic field geometry in SN 1006 and its effects on the acceleration process are therefore an open problem, and the study of thermal emission may provide useful indications, since it can be strongly affected by shock acceleration processes.

The X-ray thermal emission, compared to the non-thermal, is more uniformly distributed over the entire remnant (Rothenflug et al. 2004). Despite the importance of thermal X-rays to the study of shock acceleration processes, the physical origin of the thermal emission is still uncertain and the spatial distribution of its properties has not yet been studied.

Several studies have shown that thermal X-rays in SN 1006 cannot be uniquely associated with solar-abundance shocked plasma (e.g. Dyer et al. 2004). X-ray emitting ejecta have been observed in the north-western and south-eastern rims of the remnant (Acero et al. 2007) and even Fe K lines emission has been detected in the interior of the shell, where an iron overabundance has been revealed (Yamaguchi et al. 2008). The presence of significant X-ray emission from the shocked interstellar medium (ISM) and the value of its temperature remain controversial. Acero et al. (2007) modeled the X-ray emission of their spectral regions with one non-thermal component plus two thermal components (ejecta for the soft emission and ISM for the hard emission). However, they found that the shocked ISM component (with $k T_{\text {ISM }} \sim 1.5-2 \mathrm{keV}$ ) is almost not needed from a statistical point of view, since the quality of the fits does not change by associating the thermal emission only with the ejecta. Yamaguchi et al. (2008), instead, used three thermal components to model the $S U Z A K U$ thermal emission of very large regions of SN 1006, by associating the soft component with the shocked ISM $\left(k T_{\text {ISM }} \sim 0.5 \mathrm{keV}\right)$, and the hot components with the ejecta. Nevertheless they could not exclude that the $\mathrm{O}$ line complexes, which dominate the soft component, originate in the ejecta.

A first approach to studying the shock modification effects by the X-ray thermal emission was pursued by Cassam-Chenaï et al. (2008, hereafter C08). They focussed on the southeastern quadrant of the shell and assumed the emission in the $0.5-0.8 \mathrm{keV}$ band to be completely associated with the ejecta. They measured the ratio, $r_{\mathrm{BW} / \mathrm{CD}}$, of radii between the blast wave shock front (BW) and the contact discontinuity (CD) in several points, finding very low values with a minimum of $r_{\mathrm{BW} / \mathrm{CD}} \sim$ 1.0 near the non-thermal limbs. The azimuthal trend of $r_{\mathrm{BW} / \mathrm{CD}}$ seems to agree with the polar cap scenario, although the values of $r_{\mathrm{BW} / \mathrm{CD}}$ are too low and inconsistent with that model.
Since a spatially resolved study of the thermal emission at the rim of SN 1006 has not yet been performed, here we present the analysis of archive XMM-Newton observations of SN 1006. We focus on the rim of the remnant to study the azimuthal variations in thermal and non-thermal X-ray emission immediately behind the main shock front. We aim to obtain detailed information about the nature of the thermal emission and study its link with the shock acceleration processes. We also use a Chandra archive observation of the north-eastern limb of SN 1006 taking advantage of its larger spatial resolution to test our conclusions. The paper is organized as follows: the data and the data analysis procedure are described in Sect. 2, and in Sect. 3 we present the X-ray results in terms of spatially resolved spectral analysis and image analysis. Our results are discussed in Sect. 4 and our conclusions are summarized in Sect. 5.

\section{Data processing}

We consider all the XMM-Newton EPIC archive observations of SN 1006 available, but do not present the observation 0111090601 since it is strongly affected by proton flares and its cleaned exposure time is quite low ( $\$ 1 \mathrm{ks})$. All the observations that we analyze were performed with the Medium filter by using the Full Frame Mode for the MOS cameras (Turner et al. 2001) and the Extended Full Frame Mode for the pn camera (Strüder et al. 2001). The relevant information about the $X M M-N e w t o n$ observations presented here are summarized in Table 1. We process the data by using the Science Analysis System (SAS V7.1). Light curves, images, and spectra, were created by selecting events with PATTERN $\leq 12$ for the MOS cameras, PATTERN $\leq 4$ for the pn camera, and FLAG $=0$ for both. To reduce the contamination by soft proton flares, we screened the original event files using the sigma-clipping algorithm suggested by Snowden \& Kuntz (2007). The screened exposure times are given in Table 1.

We produced the images of the entire remnant by superimposing (using the EMOS AIC task) the MOS1, MOS2, and pn images of the pointings shown in Table 1 . We took account of the differences between MOS and pn effective areas, and the mosaiced images are MOS-equivalent. All the images are background-subtracted, vignetting-corrected, and adaptively smoothed (with the task ASMOOTH). The exposure and vignetting corrections were performed by dividing the count images by the corresponding superposed exposure maps (obtained with the task EEXPMAP).

Spectral analysis was performed in the energy band 0.5-5 keV using XSPEC. To check the validity and the robustness of our result we adopted two different procedures for spectral extraction and background subtraction.

1 - We used the event files generated with SAS. We produced the Ancillary Response Files with the SAS ARFGEN task, and used the EVIGWEIGHT task (Arnaud et al. 2001) to correct vignetting effects. The contribution of the background was derived from the high statistics background event files discussed by Carter \& Read (2007). We subtracted the background contribution from the same region positions of the CCD (i.e., in the detector coordinates), to take account of the inhomogeneous response of the instruments across the field of view. For each of the spectral regions presented in Sect. 3, we extract the spectrum from the corresponding event file with the longest exposure time. Spectra were rebinned to achieve a signal-to-noise ratio per bin $>5 \sigma$ and the fittings were performed simultaneously on 
Table 1. Relevant information about the data.

\begin{tabular}{lcccc}
\hline \hline OBS_ID & Date & MOS-pn $t_{\exp }(\mathrm{ks})^{*}$ & RA, J2000 & Dec, J2000 \\
\hline 0111090101 & $2000-08-20$ & $7-3$ & $15^{\mathrm{h}} 03^{\mathrm{m}} 50^{\mathrm{s}}$ & $-41^{\circ} 47^{\prime} 00^{\prime \prime}$ \\
0111090601 & $2001-08-08$ & $7-3$ & $15^{\mathrm{h}} 03^{\mathrm{m}} 30^{\mathrm{s}}$ & $-42^{\circ} 01^{\prime} 00^{\prime \prime}$ \\
0077340101 & $2001-08-10$ & $30-21$ & $15^{\mathrm{h}} 01^{\mathrm{m}} 51^{\mathrm{s}}$ & $-41^{\circ} 49^{\prime} 00^{\prime \prime}$ \\
0077340201 & $2001-08-10$ & $24-19$ & $15^{\mathrm{h}} 04^{\mathrm{m}} 07^{\mathrm{s}}$ & $-41^{\circ} 52^{\prime} 32^{\prime \prime}$ \\
0143980201 & $2003-08-14$ & $16-11$ & $15^{\mathrm{h}} 03^{\mathrm{m}} 30^{\mathrm{s}}$ & $-41^{\circ} 48^{\prime} 12^{\prime \prime}$ \\
0202590101 & $2004-02-10$ & $26-17$ & $15^{\mathrm{h}} 02^{\mathrm{m}} 35^{\mathrm{s}}$ & $-42^{\circ} 04^{\prime} 37^{\prime \prime}$ \\
0306660101 & $2005-08-21$ & $11-4$ & $15^{\mathrm{h}} 03^{\mathrm{m}} 35^{\mathrm{s}}$ & $-42^{\circ} 04^{\prime} 20^{\prime \prime}$ \\
\hline
\end{tabular}

* Unscreened/Screened exposure time.

both MOS spectra and on the pn spectrum. The reported errors are at $90 \%$ confidence.

2 - We used the Extended Sources Analysis Software (XMM-ESAS V2.0), which allows us to subtract instrumental and cosmic backgrounds separately. XMM-ESAS models the instrumental background from "first principles", using filter-wheel closed data and data from the unexposed corners of archived observations. The cosmic background is then modeled in XSPEC after the instrumental background subtraction. The standard XMM-ESAS method of background subtraction has some difficulties, caused by the high number of parameters and lack of suitable models for the $\mathrm{Al} \mathrm{K}_{\alpha}(\sim 1.49 \mathrm{keV})$ and $\mathrm{Si} \mathrm{K}_{\alpha}(\sim 1.75 \mathrm{keV})$ instrumental lines. To overcome these difficulties, we developed a procedure similar to the "double background-subtraction" technique (Read \& Ponman 2003). In this procedure, for each observation we produce spectra and response files from the "background" region, which is located outside the image of SN 1006. We then modeled the "background" spectra in XSPEC, and put these "background" parameters fixed when fitting "source" spectra. To improve our knowledge of local cosmic background parameters, we added to them the RASS background data ${ }^{1}$, as suggested in the XMM-ESAS manual. The obtained best-fit parameters, describing the cosmic background, remaining soft proton contribution, and instrumental lines, were then fixed and used to model the parameters of "source" regions, after proper rescaling. To prepare the EPIC MOS event lists, we used the XMM-ESAS script MOS-FILTER. We obtained the MOS1 and MOS2 spectra and constructed the corresponding background using the XMM-ESAS scripts MOS-SPECTRA and XMM-BACK, respectively. We finally grouped the spectra and the corresponding background to have at least 25 counts per bin.

We here present the results obtained with method 1 , since it allows us to use also the pn data. We verified that the results obtained are perfectly consistent with that produced with method 2 .

We also used a Chandra archive observation of SN 1006 to perform the test described in Sect. 3.2. In particular, we used the observation of the NE limb of SN1006 with ID 732, taken on 7 Jul. 2000, which has an exposure time of $69 \mathrm{ks}$.

\section{The data analysis}

\subsection{Spatially resolved spectral analysis}

Figure 1 shows the mosaiced EPIC images of SN 1006 in the $0.8-2 \mathrm{keV}$ band, $0.5-0.8 \mathrm{keV}$ band ("oxygen band", central

\footnotetext{
1 They were obtained using HEASARC background tool, http://heasarc .nasa.gov/cgi-bin/Tools/xraybg/xraybg.pl
}

panel), and in the 2-4.5 keV band ("hard band", lower panel). Since we aim to study the effects of the acceleration process, we select our spectral regions at the border of the shell. The set of 30 regions selected for the spatially resolved spectral analysis is shown (in white) in the upper panel of Fig. 1. All the regions cover the same area in the plane of the sky and they extend in the radial direction to $1.5^{\prime}$ (i.e., $\sim 10 \%$ of the SNR radius), corresponding to $\sim 1 \mathrm{pc}$ at $2.2 \mathrm{kpc}$. Our approach is similar to that followed by Rothenflug et al. (2004), although we also focus on the thermal emission.

There are striking differences between the 30 spectra. Figure 2 shows the pn and MOS spectra extracted from region 29 (upper panel), where several emission line complexes are visible, and, for comparison, from region 23 (lower panel), located in the bright north-eastern non-thermal limb, where the emission is completely featureless. Despite these differences, we plan to describe all the spectra with a unified model, so as to explain the different spectral properties in terms of azimuthal variations in the best-fit parameters. We then use an isothermal model of optically thin plasma in non-equilibrium of ionization with a linear distribution of ionization timescale versus emission measure and with free abundances (VPSHOCK model in XSPEC, Borkowski et al. 2001) to describe the thermal component, plus a synchrotron emission from an electron power law with exponential cut-off (SRCUT model in XPSEC, Reynolds \& Keohane 1999) to model the non-thermal component. We verify that the quality of the fit does not improve by adding another thermal component. Moreover, an additional thermal component introduces too many free parameters (considering the available statistics) and this generates entanglements between the best-fit values, thus determining large errors and useless results. We therefore consider only one thermal component. Our model of the thermal emission differs therefore from the two-temperatures model adopted by Acero et al. (2007) for the north-eastern and south-western regions of the shell and it is simpler than the three-temperatures model used by Yamaguchi et al. (2008), where much larger and less uniform extraction regions are considered.

We constrain the normalization of the non-thermal component (i.e., its flux at $1 \mathrm{GHz}$ ) by extracting the radio flux from the same regions defined in the X-ray map. To this end we use a radio image obtained from interferometric VLA observations with the addition of single dish data. This procedure allowed us to produce the highest angular-resolution radio image of SN 1006, together with an accurate recovery of the flux density for all spatial structures. Details on the radio image can be found in Petruk et al. (2008) (see their Sect. 3 and Fig. 3). In the fittings of the $\mathrm{X}$-ray spectra the radio flux at $1 \mathrm{GHz}$ is fixed at its best value (derived from the radio map), but we verified that allowing it to vary within its uncertainties (it is determined with a 20-50\% accuracy, depending on the spectral regions), the best-fit values and errors do not change significantly. The spectral index $\alpha$ is not 

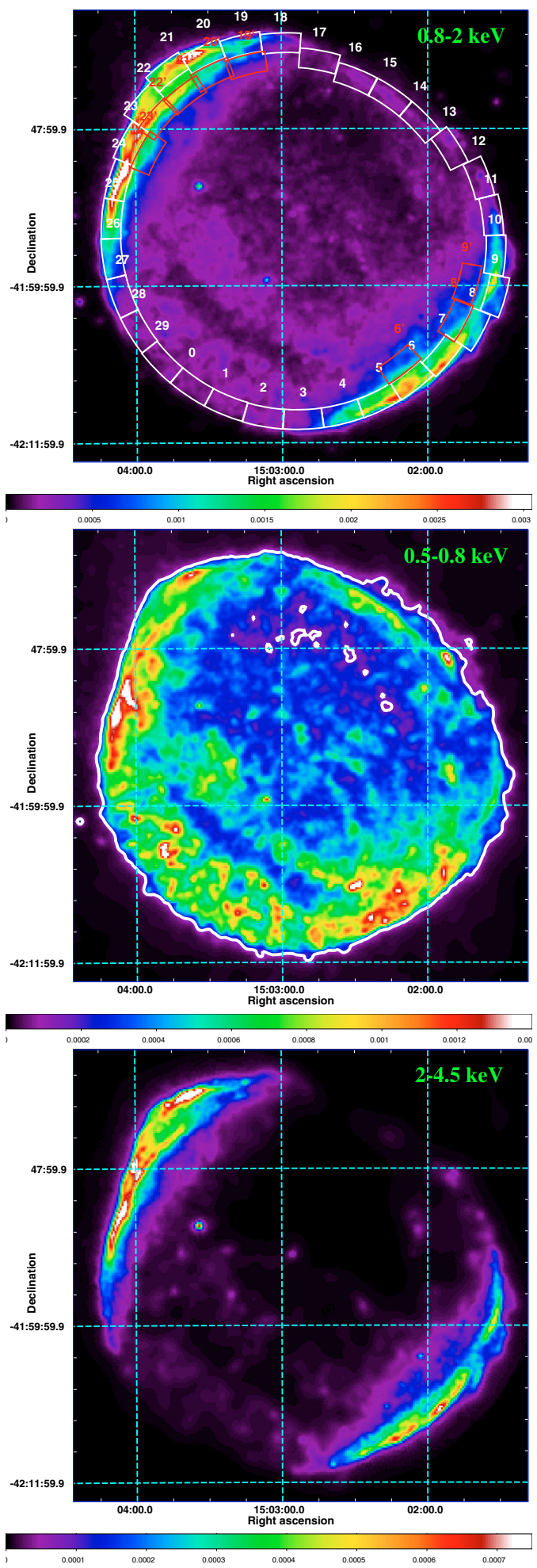

Fig. 1. Upper panel: mosaiced count-rate images (MOS-equivalent counts per second per bin) of SN 1006 in the $0.8-2 \mathrm{keV}$ band. The bin size is $8^{\prime \prime}$ and the image is adaptively smoothed to a signal-to-noise ratio 10 . The 30 regions selected for the spectral analysis of the rim (in white) and the 8 regions selected for the study of the ejecta emission measure (in red, see Sect. 3.2) are superimposed. North is up and East is to the left. Central panel: same as upper panel in the $0.5-0.8 \mathrm{keV}$ energy band. A contour level at $10 \%$ of the maximum is superimposed. Lower panel: same as upper panel in the $2-4.5 \mathrm{keV}$ energy band.

fixed in our analysis, at variance with Rothenflug et al. (2004), who fix $\alpha=0.6$.
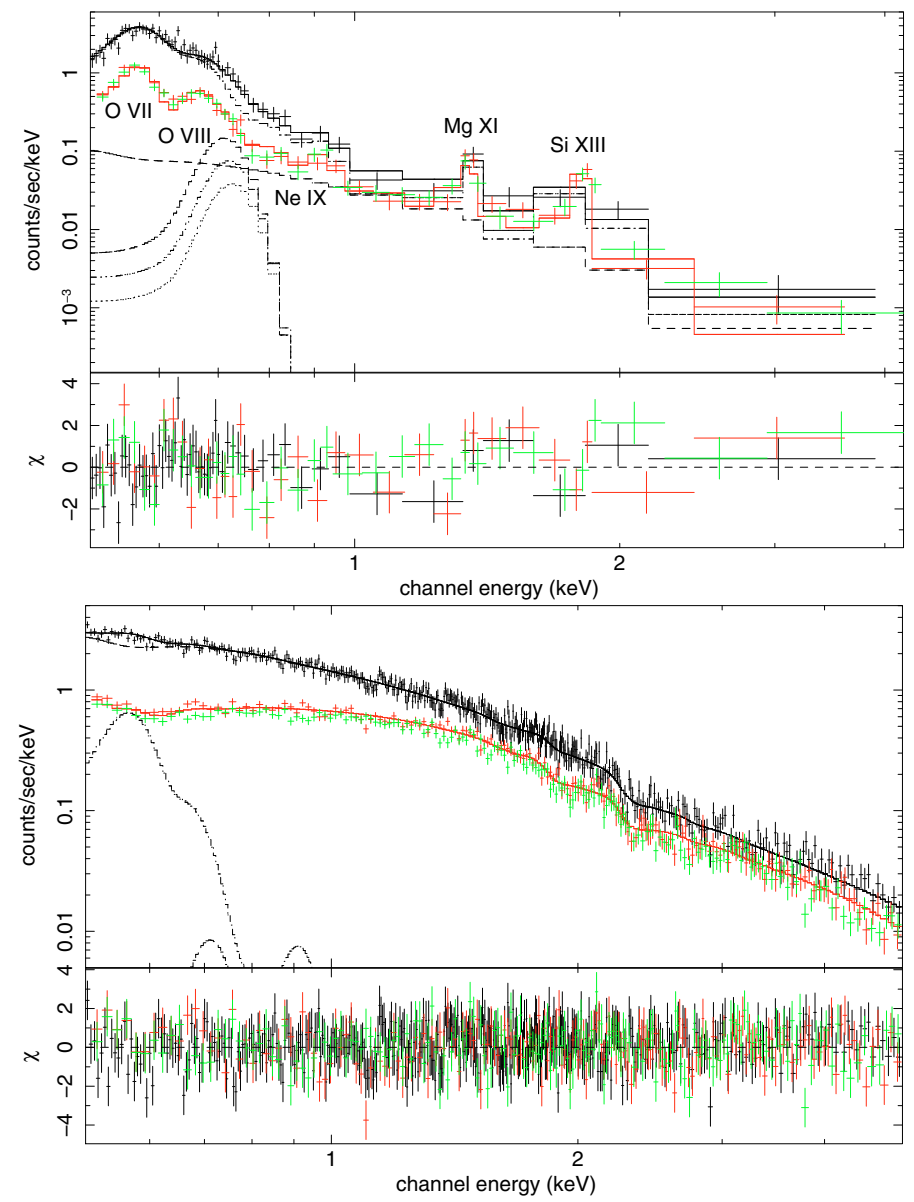

Fig. 2. Upper panel: pn spectrum (upper, in black) and MOS1, 2 (lower, in red and green) spectra of region 29 of Fig. 1 with the corresponding best-fit model and residuals. The best-fit thermal+non-thermal model described in Sect. 3.1 is shown as a continuous line, while individuals components are shown as dashed black lines for the pn spectrum only. Relevant emission lines are also indicated. Lower panel: same as upper panel for region 23 .

We find relatively large residuals between data and spectral model in the $0.7-0.85 \mathrm{keV}$ band. The same problem was observed in the $S U Z A K U$ spectra of SN 1006 by Yamaguchi et al. (2008) and interpreted as a result of the presence of K-shell O VII emission lines (transition series larger than $K_{\gamma}$ ) not included in the spectral code. To take account of the $K_{\delta}, K_{\epsilon}$, and $K_{\zeta} \mathrm{O}$ VII lines, we follow the recipe by Yamaguchi et al. (2008) and we add three narrow Gaussians (a similar procedure was also adopted by Acero et al. 2007). We fix the $N_{\mathrm{H}}$ parameter to $7 \times$ $10^{20} \mathrm{~cm}^{-2}$, in agreement with Dubner et al. (2002).

The determination of the chemical abundances is complicated by the combined presence of thermal and non-thermal continuum. We focus only on the elements whose line complexes are visible in the spectra of the "thermal" regions (i.e., $\mathrm{O}, \mathrm{Ne}, \mathrm{Mg}$, and $\mathrm{Si}^{2}$, see upper panel of Fig. 2), but we find that, even in thermal regions, the abundances are not well constrained. Although the relative ratios of $\mathrm{O}, \mathrm{Ne}, \mathrm{Mg}$, and $\mathrm{Si}$ abundances are quite well determined in each thermal region, their absolute best-fit values are entangled with the normalization of the VPSHOCK component. In particular, for a given set of best-fit abundances, we obtain similar $\chi^{2}$ values by multiplying all the abundances by the

\footnotetext{
${ }^{2}$ We fixed the other abundances to the corresponding photospheric solar values, except for the $\mathrm{S}$ abundance that is tied to that of $\mathrm{Si}$.
} 
Table 2. Best-fit parameters.

\begin{tabular}{lcc}
\hline \hline Element & Region $\mathrm{NW}^{*}$ & Region SE** \\
\hline $\mathrm{O} / \mathrm{O}_{\odot}$ & $5.0 \pm 0.1$ & $4.4 \pm 0.3$ \\
$\mathrm{Ne} / \mathrm{Ne}_{\odot}$ & $4.7_{-0.2}^{+0.1}$ & $1.5_{-0.1}^{+0.2}$ \\
$\mathrm{Mg} / \mathrm{Mg}_{\odot}$ & $12 \pm 2$ & $15 \pm 1$ \\
$\mathrm{Si} / \mathrm{Si}_{\odot}$ & $29_{-2}^{+3}$ & $50 \pm 3$ \\
\hline$k T(\mathrm{keV})$ & $0.41 \pm 0.03$ & $0.68_{-0.04}^{+0.09}$ \\
$E M^{* * *}\left(10^{17} \mathrm{~cm}^{-5}\right)$ & $1.6 \pm 0.1$ & $1.53_{-0.05}^{+0.12}$ \\
$\tau_{P S}\left(10^{10} \mathrm{~s} \mathrm{~cm}^{-3}\right)$ & $1.6 \pm 0.2$ & $1.3_{-0.3}^{+0.2}$ \\
reduced $\chi^{2}$ (d. o. f. $)$ & $1.85(426)$ & $1.84(234)$ \\
\hline
\end{tabular}

* Union of regions 13-16 of Fig. 1; ** union of regions 28, 29, 0, and 1 of Fig. 1; *** emission measure per unit area.

same factor $k$ (even with $k=100$ ) and by reducing the normalization by the same factor. To avoid this degeneracy (which also hampers the determination of the emission measure), we consider two large regions: the first one is the sum of regions 28, 29, 0 , and 1 (region SE); the second one is the sum of regions 13-16 (region NW). The higher statistics in these large regions allow us to accurately determine the chemical abundances, whose bestfit values are shown in Table 2. Notice that in the SE region the chemical composition of the X-ray emitting plasma is quite different from that in the NW region, where we find a lower $\mathrm{Si} / \mathrm{O}$ ratio. In both cases, however, we observe significant overabundances. Moreover, the high $\mathrm{Si} / \mathrm{O}$ ratios obtained in the $\mathrm{SE}$ and NW regions are typical of type Ia SNe. We therefore conclude that the thermal component is associated with shocked ejecta. We assume regions 8-22 to have the same abundances as region NW and the remaining 15 regions to have the same abundances as region SE. The results of our spectral analysis are summarized in Fig. 3.

\subsubsection{Non-thermal emission}

As shown in Fig. 3, we find that the azimuthal profile of the synchrotron cut-off frequency $v_{\text {break }}$ presents a trend that is perfectly consistent with that derived by Rothenflug et al. (2004), the cutoff frequency increasing in the non-thermal limbs. However, our break frequencies are systematically lower than their ones (by a factor of $\sim 10$ ). This discrepancy is due to the value of the radio spectral index $\alpha$. In fact, we here derive $\alpha \sim 0.50 \pm 0.04$ from our spectral fittings in the non-thermal regions ${ }^{3}$, while Rothenflug et al. (2004) do not measure $\alpha$, and assume $\alpha=0.6$. The lower values of the spectral index naturally imply lower cut-off frequencies. Notice that, as shown by the green points in Fig. 3, we obtain also $\alpha \sim 0.5$ by using the "ESAS approach" for the analysis of the background, as described in Sect. 2. Our best-fit value $\alpha \sim 0.5$ and our cut-off frequencies are in agreement with the results obtained by Allen et al. (2008).

\subsubsection{Thermal emission}

Figure 3 clearly shows that the emission measure per unit area, $E M$, of the thermal component significantly decreases in the bright non-thermal limbs (we obtain the same trend by also analyzing the spectra with the ESAS method described in Sect. 2). In the North-East (regions 19-23) and at South-West (regions 6-10), in particular, EM is a factor of $\gtrsim 4$ lower than in the North-West or South-East, and in regions 9 and 19 it is

${ }^{3}$ In the thermal regions we therefore fix $\alpha=0.5$, as shown in Fig. 3.

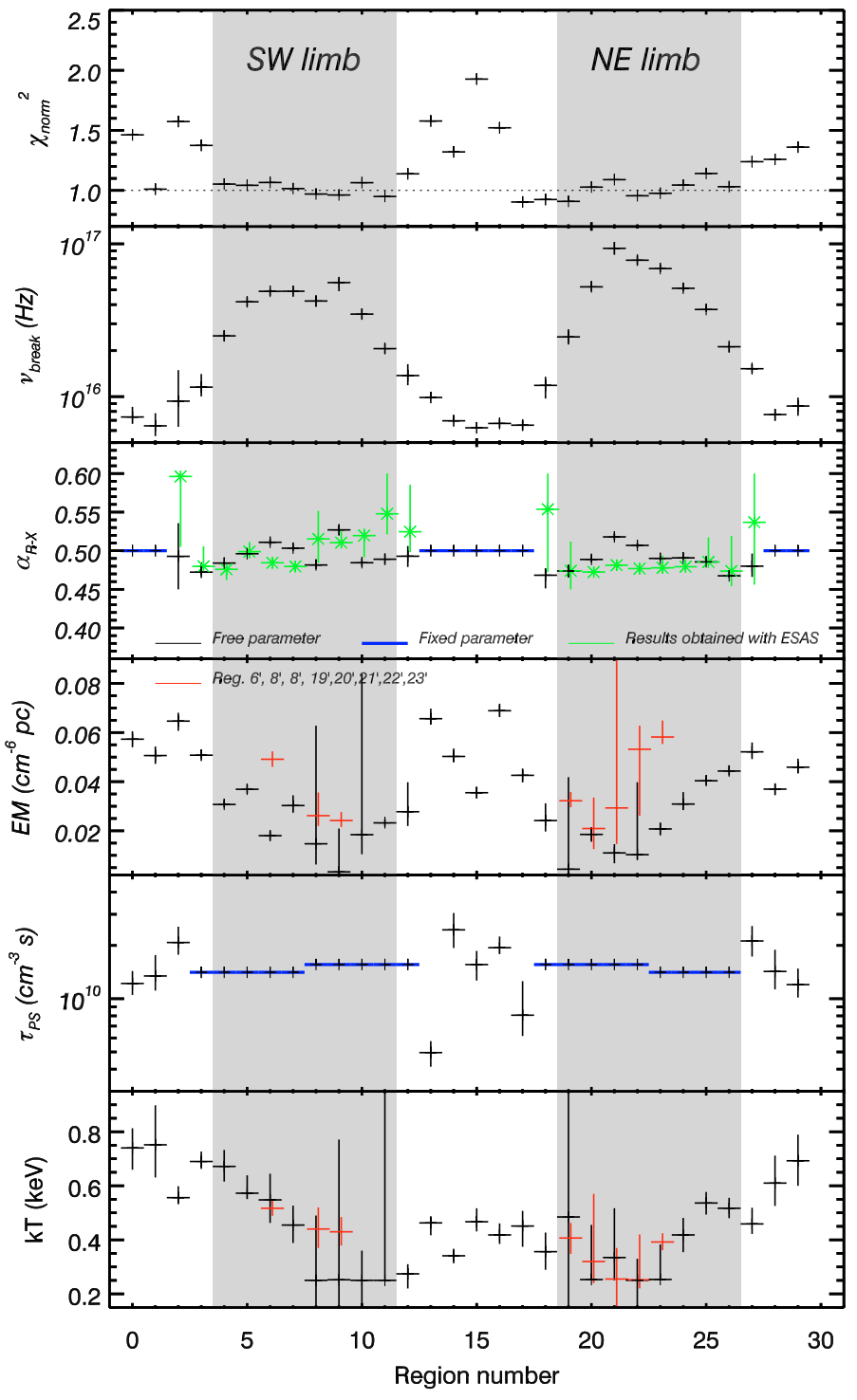

Fig. 3. Best-fit parameters for the 30 regions shown in the upper panel of Fig. 1 (in black) and for the 8 regions of the upper panel of the same figure (in red). The model consists in a non-equilibrium of ionization isothermal component plus a synchrotron SRCUT model. The blue crosses indicate where the parameters are fixed. The errors are at $90 \%$ confidence level. The chemical abundances are tied to those of region NW, for regions 8-22, and to those of region SE, for regions 23-7 (see Table 2). For comparison, the best-fit values of $\alpha$ obtained by using the "ESAS approach" for the analysis of the background, as described in Sect. 2 are shown as green stars.

consistent with being zero. This is quite surprising because one would expect $E M$ to be large in the non-thermal limbs, where the particle acceleration is supposed to be more efficient. In fact, Decourchelle et al. (2000) demonstrated that, if the main shock is modified, the post-shock density is higher, even behind the reflected shock (and this is our case, since we are observing the shocked ejecta). We investigate in detail the reason and the physical origin of this result in the next section.

The temperature of the ejecta is not uniform and in the northwestern part of the rim, in particular (regions 13-18 in Fig. 3), the temperatures are clearly lower. Unfortunately, in the nonthermal limbs, the determination of temperature is affected by large errors, because of the low EM. 
The ionization time scale $\tau_{\text {PS }}$ was determined only in thermal regions and is quite uniform, with a value of around $10^{10} \mathrm{~s} \mathrm{~cm}^{-3}$ (in the non-thermal regions we fixed it to its mean value in the adjacent thermal regions). We observe a large deviation from the average value only in region 13 , where $\tau_{\mathrm{PS}}=5.0_{-0.9}^{+0.8} \times$ $10^{9} \mathrm{~s} \mathrm{~cm}^{-3}$. This is not surprising because region 13 contains the bright oxygen knot studied in detail by Vink et al. (2003), who found $\tau_{\mathrm{NEI}} \sim 2.35 \pm 0.07 \times 10^{9} \mathrm{~s} \mathrm{~cm}^{-3}$. Vink et al. (2003) used the NEI model (while we use the VPSHOCK model) and their value should be compared with $\tau_{\mathrm{PS}} / 2$ (see Borkowski et al. 2001), and our estimates are therefore perfectly consistent with theirs. We also note that the relative abundances found by Vink et al. (2003) in the oxygen knot of ejecta are quite similar to those we derive for region SE, namely Vink et al. (2003) find $\mathrm{O}: \mathrm{Ne}: \mathrm{Mg}: \mathrm{Si} \sim 0.18: 0.05: 0.2: 1$ in the oxygen knot, and we find O:Ne:Mg:Si 0.1:0.03:0.3:1 in region SE (see Table 2). This result confirms further that the thermal component is associated with the ejecta.

\subsection{X-ray emitting ejecta in SN 1006}

Since the thermal emission is completely dominated by the shocked ejecta, it is interesting to study their spatial distribution and obtain an image of their X-ray emission. Bamba et al. (2008) suggested that there are differences in the spatial distribution of thermal and non-thermal emission in the north-eastern region of SN 1006. Cassam-Chenaï et al. (2008) assumed that (in the south-eastern quarter of the shell) the X-ray emission in the "oxygen band" $(0.5-0.8 \mathrm{keV})$ is completely associated with the ejecta. Indeed, even in this soft band, there is a non-negligible flux associated with the non-thermal component, especially in the non-thermal limbs (see Fig. 2). We use our results for the non-thermal component to estimate this contribution.

The spectral properties of the SRCUT component are very robust and reliable. They do not depend strongly on the model adopted for the thermal component, since the normalization of the synchrotron emission is derived from the radio data and the values of $\alpha$ and the azimuthal profile of the roll-off frequency are in good agreement with those reported in literature. Using our result, we calculate that in only one third of the rim (regions 28-2 and 18-22) the contribution of the thermal emission in the oxygen band is larger than $80 \%$ of the total flux, while in fifteen regions (4-11 and 19-25) it is $\leq 50 \%$. This means that the $0.5-0.8 \mathrm{keV}$ map of SN 1006 shown in the central panel of Fig. 1 cannot be used as a proxy of the ejecta.

The procedure that we use to produce the image of the "pure" thermal emission in the oxygen band from our spectral results on the non-thermal emission is described in detail in Appendix A and is based on the assumption that the emission in the hard band $(2-4.5 \mathrm{keV})$ is entirely non-thermal (as for the validity of this assumption, see Appendix A). With this assumption, it is possible to use the spectral results to extrapolate the non-thermal emission in the oxygen band from the hard-band image (in Appendix, we show that the effects of the uncertainties in the best-fit parameters are negligible). Then, by subtracting the non-thermal contribution, we obtain the pure thermal image (i.e., the emission of the shocked ejecta) in the $0.5-0.8 \mathrm{keV}$ band. The map is shown in Fig. 4 and presents striking differences from the total image in the same band (central panel of Fig. 1), especially in the nonthermal limbs, where the emission of the ejecta is negligible.

In particular, in the spectral regions where the emission measure of the thermal component is low (e.g., regions 8, 9, 19, 21, 22, see Fig. 3), the thermal image indicates that the filling

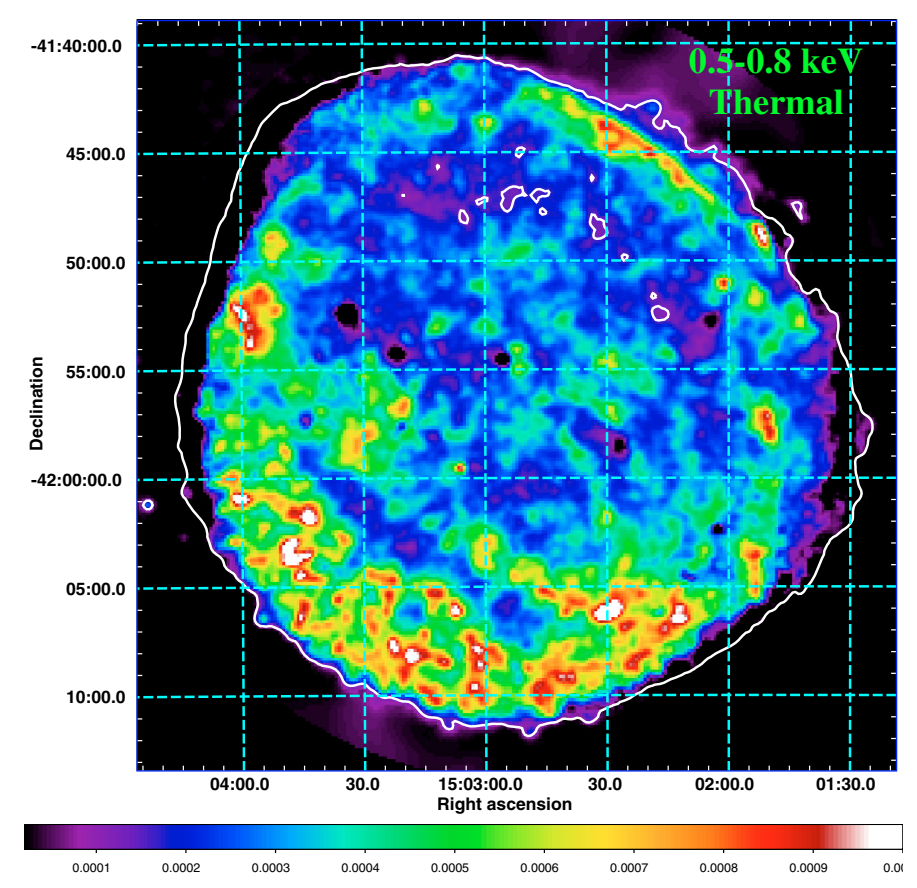

Fig. 4. Mosaiced pure thermal image (MOS-equivalent counts per second per bin) of SN 1006 in the $0.5-0.8 \mathrm{keV}$ band. The contribution of the non-thermal emission in the soft energy band has been subtracted. The bin size is $8^{\prime \prime}$ and the image is adaptively smoothed to a signalto-noise ratio 10 . The same contour level shown in the central panel of Fig. 1 is overimposed for comparison.

factor, $f$, of the ejecta is at its minimum. Therefore, it is the low volume filled by the X-ray emitting thermal plasma (in the spectral region) that causes the decrease in the emission measure. The comparison between Figs. 3 and 4 reveals, in general, that there is a clear relationship between $f$ and $E M$ : the variations in the emission measure of the thermal X-ray component can be associated with the different filling factors of the ejecta in the spectral regions.

To verify that this conclusion is correct and that our map of the soft emission of the ejecta is reliable, we perform two tests:

1 - We consider 8 regions that, according to our thermal map, are expected to contain a larger filling factor of ejecta than regions $19-23$ and 6,8 , and 9. These new regions are indicated (in red) in the upper panel of Fig. 1. Figure 3 shows that the $E M$ in these new regions (indicated by the red crosses) is significantly higher than that of the corresponding regions on the rim, as predicted by our thermal image. Notice that the extension along the line of sight in these new regions is only a factor $\$ 10 \%$ higher than in regions $19-23$ and 6,8 , and 9 , and therefore the much larger variations in $E M$ are, indeed, due to higher ejecta filling factors.

2 - We exploit the high Chandra spatial resolution to extract Chandra ACIS-I spectra from the very narrow regions shown in Fig. 5. Region A covers a thin non-thermal filament, region B is immediately behind, where the emission in the $0.5-0.8 \mathrm{keV}$ band remains high, but is not associated with the ejecta, according to our thermal image, while region $C$ covers a bright knot in our thermal image of the ejecta. The spectra extracted from these regions are shown in the lower panel of Fig. 5 and confirm the predictions of our thermal image: no O-line emission is visible in the spectrum of region $\mathrm{B}$, which is very similar to that of the 


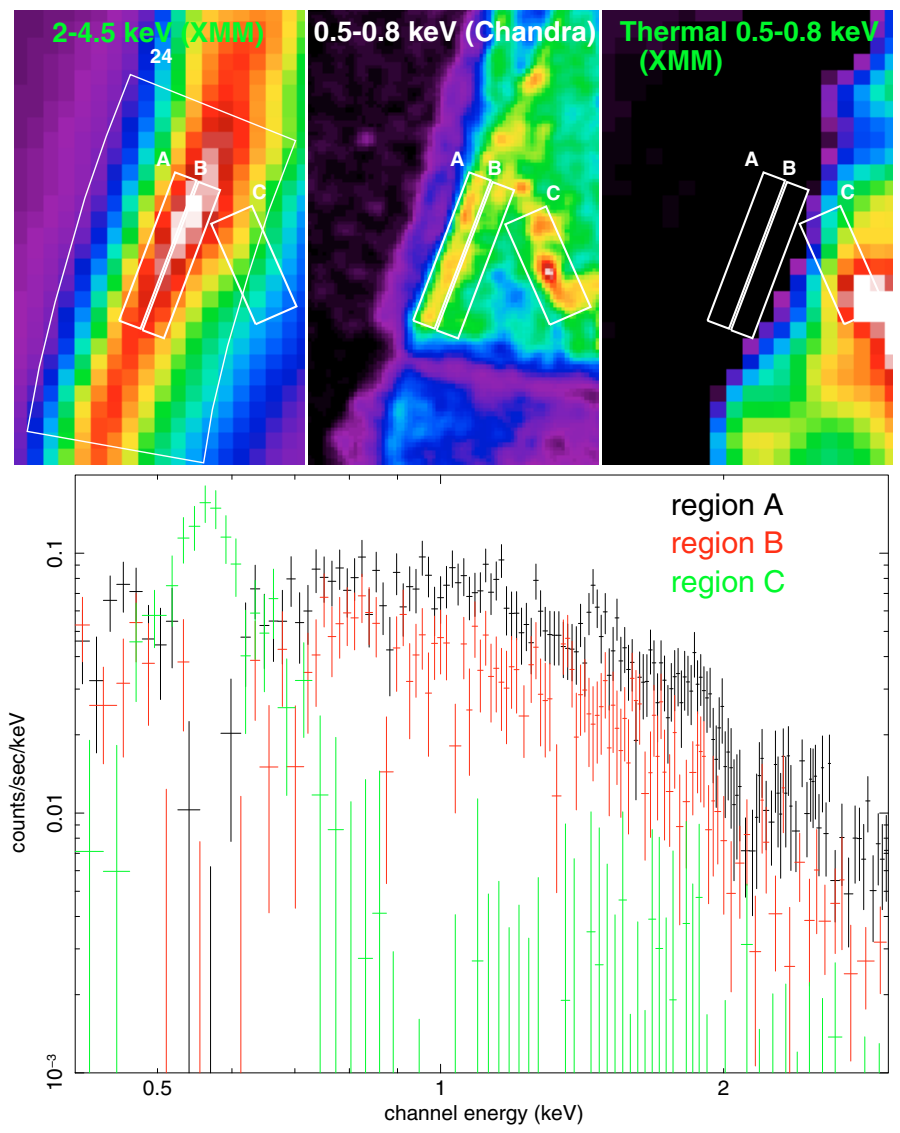

Fig. 5. Upper panel: close-up view of region 24 of Fig. 1. The $X M M$-Newton count-rate images in the hard band is shown, together with the Chandra ACIS-I image in the $0.5-0.8 \mathrm{keV}$ band compared with our XMM-Newton thermal image in the same band (Fig. 4). The three boxes indicate the regions where we extracted the Chandra ACIS spectra. Lower panel: Chandra ACIS spectra extracted from the three regions shown in the upper panel.

non-thermal region $\mathrm{A}$, while in region $\mathrm{C}$ the oxygen line is clearly visible.

\section{Discussion}

\subsection{Properties of the ejecta}

Our spatially resolved spectral analysis shows that thermal X-ray emission can be completely associated with the ejecta and the high $\mathrm{Si} / \mathrm{O}$ ratios obtained in the SE and NW regions are typical of type Ia SNe. Despite the overall spherical symmetry of the remnant, the distribution of the ejecta in the shell is not uniform. In particular, we detect inhomogeneities in the chemical composition of the ejecta, with the south-eastern part of the rim characterized by a lower $\mathrm{Si}$ abundance and a higher Ne abundance than the north-western part. We also observe lower ejecta temperatures in the north-western part of the rim in agreement with the findings of Acero et al. (2007). The interpretation of this result is not straightforward. As proposed by Acero et al. (2007), in the north-west the remnant is interacting with a dense medium, and produces a very bright $\mathrm{H}_{\alpha}$ filament (Winkler et al. 2003), which is spatially correlated with the X-ray thermal emission. The physical conditions then differ from that in the southeast, where the expansion of the shell occurs in a more uniform medium. It is possible that the different thermal conditions between NW and SE are due to different heating mechanisms: we can in particular suppose that the ejecta are heated by the reverse shock at SE and by a reflected shock, generated by the interaction of the main shock front with the density enhancement, at NW. According to this scenario, the ISM density should be lower behind the dense cloud, thus causing an almost free expansion in that direction until the shock has reached the dense $\mathrm{H}_{\alpha}$ cloud and generated the reflected shock. This may also explain the differences in the abundances of the ejecta, since it is possible that the reflected and reverse shock originated at different epochs (the interaction with the dense cloud is relatively recent) and have, therefore, interacted with different layers of the expanding ejecta. Nevertheless, according to this scenario, we would expect to measure different ionization time scales in the two regions, while we find that the ionization time scales at NW are quite similar to those at SE (see Table 2 and Fig. 3) and this seems to indicate that the inhomogeneities in the abundances are intrinsic (i.e., are the result of an anisotropic explosion) and not due to differences between the reverse and the reflected shock.

We can estimate the time elapsed after the shock impact, $t$, and the ejecta density $n_{\mathrm{ej}}$. We determine $t$ from our best-fit values of $E M$ and $\tau_{\mathrm{PS}}$ in the large NW and SE regions (Table 2). To this end we first derive the post-shock density of the ejecta from their emission measure per unit area. We first consider the NW and $\mathrm{SE}$ regions and, by assuming that the extension along the line of sight is $L \sim 1.85 \times 10^{19} \mathrm{~cm}$ (i.e., the length of the chord intercepted by the shell along the line of sight at the center of these spectral regions), we derive the density $n_{\mathrm{ej}} \sim 0.1 / \sqrt{f} \mathrm{~cm}^{-3}$ (where $f$ is the filling factor of the ejecta) both at NW and SE. We perform the same estimate in each of the thermal regions (regions 0-2, 13-17, and 27-29) to check for the presence of azimuthal variations in the density, but we do not find any evidence for it. We then derive the time elapsed after the shock impact in the NW and SE regions, by using the relationship: $t \sim \tau_{\mathrm{PS}} / n_{\mathrm{ej}} \sim 2500 \pm 500 \sqrt{f}$ yr. Our value of $t$ is reasonable, considering that $f<1$, because of the clumpy nature of the ejecta.

\subsection{The distance between contact discontinuity and blast wave shock}

The image of the pure thermal emission of the ejecta, shown in Fig. 4, allows us to trace the position of the contact discontinuity, CD, over the whole remnant. The distance of the CD from the blast wave position, BW, can be a powerful tracer of efficient particle acceleration at the blast wave shock. Decourchelle et al. (2000) showed that, when the injection efficiency $\eta$ increases, the distance between BW and CD decreases. Rothenflug et al. (2004) showed that the non-thermal X-ray emission of SN 1006 is consistent with the polar cap scenario and this implies that $\eta$ is higher in the non-thermal limbs, where, therefore, we expect the contact discontinuity to be closer to the shock front. C08 measured the distance between BW and CD in the southeastern quadrant of the shell, finding that the ratio BW/CD decreases towards the non-thermal limbs, as expected, but reaches too low values $(\sim 1.0)$. Nevertheless, to derive the position of the contact discontinuity, they assumed that the emission in the $0.5-0.8 \mathrm{keV}$ band is a tracer of the ejecta, and we verified that this assumption is valid only in a small portion of the shell. We therefore adopt the same diagnostics used by $\mathrm{C} 08$, but we here use our pure thermal image in the oxygen band (Fig. 4) to trace the contact discontinuity over the shell.

We consider the radial profiles of the surface brightness in our thermal image for 360 different angles (the azimuthal angle 


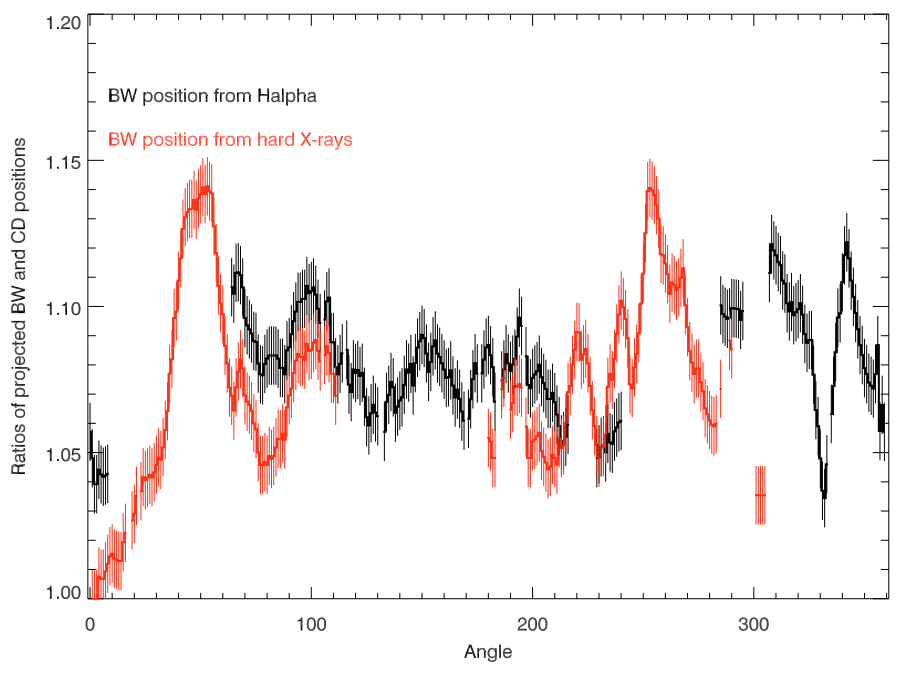

Fig. 6. Azimuthal profile of the ratio of the position of the blast wave shock to the contact discontinuity with corresponding error bars. In order to reduce the fluctuations, for each angle we plot the moving average of the ratio, computed considering the ten adjacent points. The position of the contact discontinuity is derived from the pure thermal image in the oxygen band shown in Fig. 4, while the position of the blast wave shock is derived from the $\mathrm{H}_{\alpha}$ image of $\mathrm{SN} 1006$ presented in Winkler et al. (2003) (black curve, missing points mark locations where $\mathrm{H}_{\alpha}$ filaments are not well defined) and from the hard X-ray image shown in the lower panel of Fig. 1 (in red). The angle is measured counterclockwise from the North.

increases counterclockwise from the North direction) and, for each angle, we identify the $\mathrm{CD}$ as the position of the relative maximum in brightness at the largest distance from the center ${ }^{4}$, having a surface brightness larger than a given threshold. We fix the threshold to be $10 \%$ of the flux at the brightest point in the pure thermal image. Since the pixel-size of the thermal map is $\sim 50 \%$ of the EPIC point-spread function (FWHM), this procedure provides a quite accurate estimate of the CD position. The associated error is the addition in quadrature of the pixel size $\left(8^{\prime \prime}\right)$ and of the standard deviation of the Gaussian function used to smooth the image (one pixel). The position of the main shock front in the thermal regions can be derived from the deep $\mathrm{H}_{\alpha}$ image presented in Winkler et al. (2003), corrected for the remnant expansion in the years elapsed from the optical observation to our observations (assuming an expansion speed of $4000 \mathrm{~km} \mathrm{~s}^{-1}$ ). As explained by C08, it is difficult to identify the optical rim by using a quantitative criterion (e.g., a threshold in the radial profile), so we determine the location of the BW, by inspecting by eye the optical image. Nevertheless, it is impossible to use the $\mathrm{H}_{\alpha}$ image to trace the position of the blast wave shock in the non-thermal limbs, since the optical emission is quite noisy and does not present sharp features. Therefore, in these regions, we use the $2-4.5 \mathrm{keV}$ image (lower panel of Fig. 1) to trace the position of the blast wave shock, by adopting the same criterion as that used for the CD.

Figure 6 shows the azimuthal profile of the ratio of $\mathrm{BW}$ to $\mathrm{CD}, r_{\mathrm{BW} / \mathrm{CD}}$, over the entire shell. In particular, to reduce the noise level, we plot for each angle the moving average of $r_{\mathrm{BW} / \mathrm{CD}}$ computed by considering the ten adjacent points. By comparing our azimuthal profile of $r_{\mathrm{BW} / \mathrm{CD}}$ with that obtained in the southeastern quadrant by $\mathrm{C} 08$, we notice that, although remarkable agreement in the thermal regions is present, our $r_{\mathrm{BW} / \mathrm{CD}}$ does not

\footnotetext{
4 The positions of the blast wave shock and of the contact discontinuity are measured with respect to the same center as that chosen by $\mathrm{C} 08$.
}
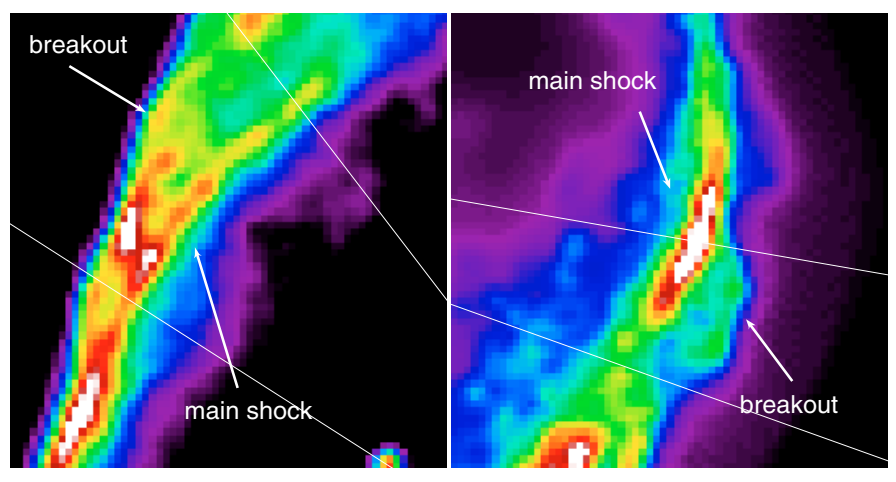

Fig. 7. Close-up view of the $2-4.5 \mathrm{keV}$ band image of Fig. 1 showing two shock breakouts in the rim of SN 1006. We superimpose two lines indicating the azimuthal angles 40 and 60 (left panel) and 250 and 260 (right panel).

Table 3. Parameters of the 3D MHD model.

\begin{tabular}{ll}
\hline \hline ISM density & $0.05 \mathrm{~cm}^{-3}$ \\
Ambient magnetic field & $30 \mu \mathrm{G}$ \\
Explosion energy & $1.3 \times 10^{51} \mathrm{erg}$ \\
Mass of the ejecta & $1.4 M_{\odot}$ \\
\hline Shock speed after $1000 \mathrm{yr}$ & $4750 \mathrm{~km} \mathrm{~s}^{-1}$ \\
Shell radius after $1000 \mathrm{yr}$ & $8.65 \mathrm{pc}$ \\
\hline
\end{tabular}

decrease to very low values near the non-thermal limbs (at odds with $\mathrm{C} 08$, who finds $r_{\mathrm{BW} / \mathrm{CD}} \sim 1.0$ ). This is because the pure thermal image in the oxygen band differs significantly from the total $0.5-0.8 \mathrm{keV}$ image close to the non-thermal limbs. The value of $r_{\mathrm{BW} / \mathrm{CD}}$ is quite uniform and does not present relevant azimuthal variations, except for a couple of regions (between $\sim 40$ and $\sim 60$ degrees, and between $\sim 250$ and $\sim 260$ degrees) where it reaches two maxima at $\sim 1.15$. We verify that in these two regions remarkable shock breakouts are present, as shown in Fig. 7. We interpret these breakouts as the result of the propagation of the main shock in a locally rarefied medium. If the shock has recently encountered a low density inhomogeneity its speed is larger with respect to the adjacent regions, while the position of both the reverse shock and the contact discontinuity are not immediately affected. This explains the locally high values of $r_{\mathrm{BW} / \mathrm{CD}}$. Elsewhere $r_{\mathrm{BW} / \mathrm{CD}}$ is limited to within the range $r_{\mathrm{BW} / \mathrm{CD}} \sim 1.05-1.12$.

We verify that this value is not consistent with that expected for a non-modified Sedov shock. To this end we consider the 3D magnetohydrodynamic model developed by Orlando et al. (2007) to describe the expansion of a SNR through the magnetized ISM, adopting a set of parameters appropriate for SN 1006 (see Table 3). The model includes no eventual magnetic-field amplification, and no effects on shock dynamics due to backreaction of accelerated cosmic rays. We explore different configurations of the interstellar magnetic field by performing a set of numerical simulations using FLASH, an adaptive meshrefinement multiphysics code (Fryxell et al. 2000). In all the cases we find that Richtmyer-Meshkov (R-M) instability develops in the inner shell, as the forward and reverse shocks progress through the ISM and ejecta, respectively (e.g., Kane et al. 1999). After $1000 \mathrm{yrs}$ of evolution, the R-M instability determines a quite complex 3D density and temperature structure of the contact discontinuity which extends a bit further with respect to the unidimensional case (where the instabilities are not described), leading to different values of the ratio $r_{\mathrm{BW} / \mathrm{CD}}$. The results of our 


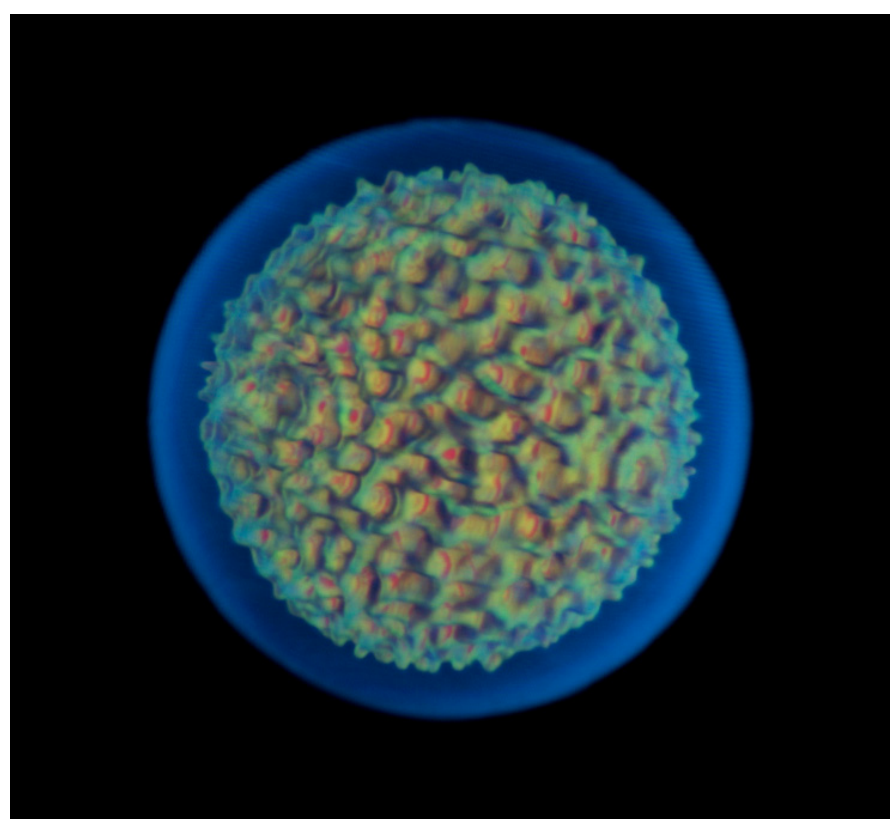

Fig. 8. 3D rendering of an MHD simulation describing the expansion of SN1006 through a magnetized ISM at $t=1000 \mathrm{yrs}$ (the parameters of the model are shown in Table 3). The ejecta material is tracked with a "solid" surface, the shocked ISM in semi-transparent blue.

simulation at $t=1000$ yrs are shown in Fig. 8: the "solid" surface is a tracer of the ejecta material, while the density of the shocked ISM is shown in semi-transparent blue. In all the cases examined, we find that, according to our MHD model of a nonmodified SNR shock, we expect $r_{\mathrm{BW} / \mathrm{CD}}>1.16$, at $t=1000 \mathrm{yrs}$. As shown in Fig. 6, we observe much lower values of $r_{\mathrm{BW} / \mathrm{CD}}$ in SN 1006. As shown by Decourchelle et al. (2000) and Ellison et al. (2004), when efficient particle acceleration is present and a significant fraction of the shock energy is deposited in cosmic rays, the region between $\mathrm{BW}$ and $\mathrm{CD}$ becomes narrower, thus making $r_{\mathrm{BW} / \mathrm{CD}}$ lower. Since we observe much lower values of $r_{\mathrm{BW} / \mathrm{CD}}$ in the whole rim with respect to the non-modified case, we conclude that the shock in SN 1006 is modified everywhere.

If the ambient magnetic field were aligned in the SW-NE direction in the plane of the sky, and had no component along the line of sight, we would expect lower values of $r_{\mathrm{BW} / \mathrm{CD}}$ in the nonthermal limbs, at odds with our findings. Nevertheless, if the aspect angle, $\phi$, between the interstellar magnetic field and the line of sight differed significantly from a right angle (i.e., the interstellar magnetic field is not in the plane of the sky and $\phi \lesssim 70$ degrees), we would not observe significant variations in $r_{\mathrm{BW} / \mathrm{CD}}$, since, in this case, we would not observe the region where the acceleration process is more efficient edge-on. Therefore, our results suggest that the aspect angle in SN 1006 differs significantly from $90^{\circ}$. A similar conclusion was obtained by Petruk et al. (2008) by comparing observed and synthesized radio maps of SN 1006.

\section{Summary and conclusions}

Our study of the X-ray emission of SN 1006 has shown that:

1. The emission at the rim of the shell can be described by a mixture of thermal emission from plasma in non-equilibrium of ionization and non-thermal SRCUT emission. We compare two different approaches to X-ray spectra analysis, obtaining perfectly consistent results with the two methods.
2. We measure the radio-to-X-ray photon index of the nonthermal component, finding $\alpha \sim 0.5$, and we confirm the azimuthal profile of the roll-off frequency found by Rothenflug et al. (2004), revising the frequency values in the light of the new value of $\alpha$.

3. Thermal emission can be associated with shocked ejecta. The temperature and the chemical composition of the ejecta are not uniform. In particular, we find lower temperatures and lower $\mathrm{Si} / \mathrm{O}$ abundances in the NW than the SE.

4. Our pure thermal image in the $0.5-0.8 \mathrm{keV}$ band shows that even in this soft band, the emission at the bright limbs is mainly associated with the non-thermal component.

5. We derive the azimuthal profile of the ratio $r_{\mathrm{BW} / \mathrm{CD}}$ between the position of the blast wave shock and the contact discontinuity in the whole shell. We find that $r_{\mathrm{BW} / \mathrm{CD}}$ is fairly uniform and its mean value is lower than that expected for a non-modified shock, even taking into account hydrodynamic instabilities that cause $r_{\mathrm{BW} / \mathrm{CD}}$ to be lower than that derived from simple 1D hydrodynamic models. We conclude that the shock is modified everywhere and that the aspect angle differs significantly from $90^{\circ}$.

Acknowledgements. We wish to thank F. Winkler for providing us the $\mathrm{H}_{\alpha}$ image in digital format. This work makes use of results produced by the PI2S2 Project managed by the Consorzio COMETA, a project co-funded by the Italian Ministry of University and Research (MIUR) within the Piano Operativo Nazionale "Ricerca Scientifica, Sviluppo Tecnologico, Alta Formazione" (PON 2000-2006). More information is available at http://www.pi2s2.it and http://www. consorzio-cometa.it. The software used in this work was in part developed by the DOE-supported ASC/Alliance Center for Astrophysical Thermonuclear Flashes at the University of Chicago. ESAS calculations were performed on computational resources of BITP computing cluster and VIRGO.UA Virtual Observatory. The work of D.I. was supported by the Program of Fundamental Research of the Physics and Astronomy Division of the National Academy of Sciences of Ukraine. D.I. and O.P. acknowledge the program "Kosmomicrofizyka" of the National Academy of Sciences of Ukraine. I.T. acknowledges the support from the INTAS YSF grant No. 06-1000014-6348. I.T. and D.I. thank the Faculty of the European Space Astronomy Centre (ESAC), where a part of this work was done, for financial support. G.D. and G.C. are members of CONICET (Argentina). The project was partially funded by grants from CONICET, UBA and ANPCYT (Argentina).

\section{Appendix A: Production of the pure thermal image}

We here describe the procedure we developed to produce the image of the thermal emission in the $0.5-0.8 \mathrm{keV}$ band (we call this image $T H$ ). The scheme of the procedure consists of three steps: A) we assume the emission in the $2-4.5 \mathrm{keV}$ band to be completely associated with the non-thermal component; B) we extrapolate the $2-4.5 \mathrm{keV}$ image to produce an image of the non-thermal component in the $0.5-0.8 \mathrm{keV}$ band (we call this image $N O N T H$ ) by using the results of our spatially resolved spectral analysis; and C) we subtract NONT H from the total image in the $0.5-0.8 \mathrm{keV}$ band, thus obtaining the thermal image. In the following, we describe in detail these three steps.

\section{A.1. Step A (assumption)}

We assume the thermal emission to be negligible in the $2-4.5 \mathrm{keV}$ band. The results of our spatially resolved spectral analysis show indeed that this assumption is strictly valid in the non-thermal limbs (where the non-thermal flux is $\gtrsim 99 \%$ of the total), while it does not hold in the thermal regions (e.g. regions $28-2$ ), where about $50 \%$ of the flux in the $2-4.5 \mathrm{keV}$ is associated with the thermal component. Nevertheless, we can show that this problem does not generate significant effects. In fact, even if we overestimate the contribution of the synchrotron 

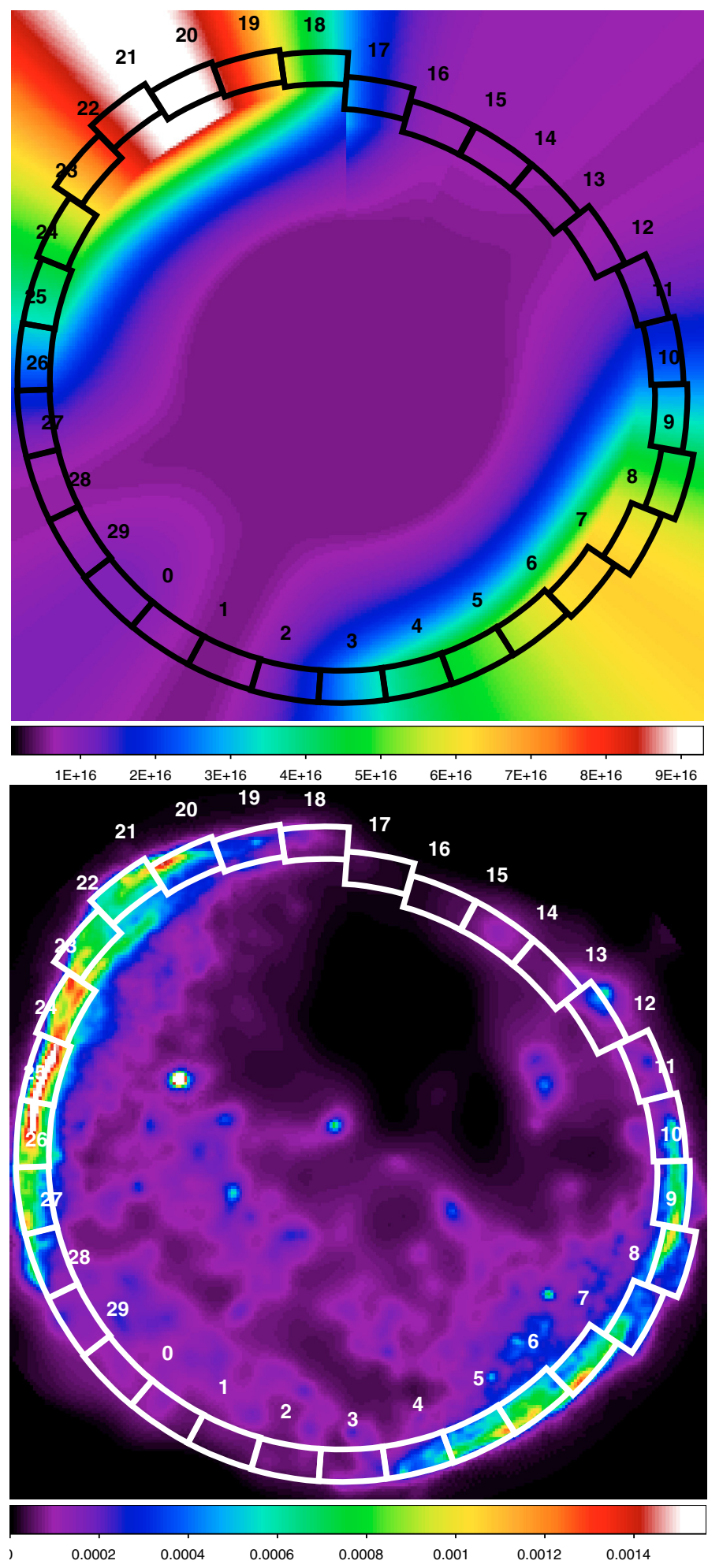

Fig. A.1. Upper panel: map of the cut-off frequency derived according to the procedure described in Sect. A.2. The 30 regions selected for the spectral analysis of the rim are indicated in black. Lower panel: image of the non-thermal emission in the $0.5-0.8 \mathrm{keV}$ band.

emission in the thermal region (by a factor of a few) this contribution remains small in the $0.5-0.8 \mathrm{keV}$ band: for example we verified (after step B) that it is $\sim 10 \%$ and $\sim 6 \%$ of the total flux in the oxygen band in region 0 and region 16 , respectively. This means that, when we produce $T H$, the flux in the thermal regions is not significantly underestimated, while that in the non-thermal

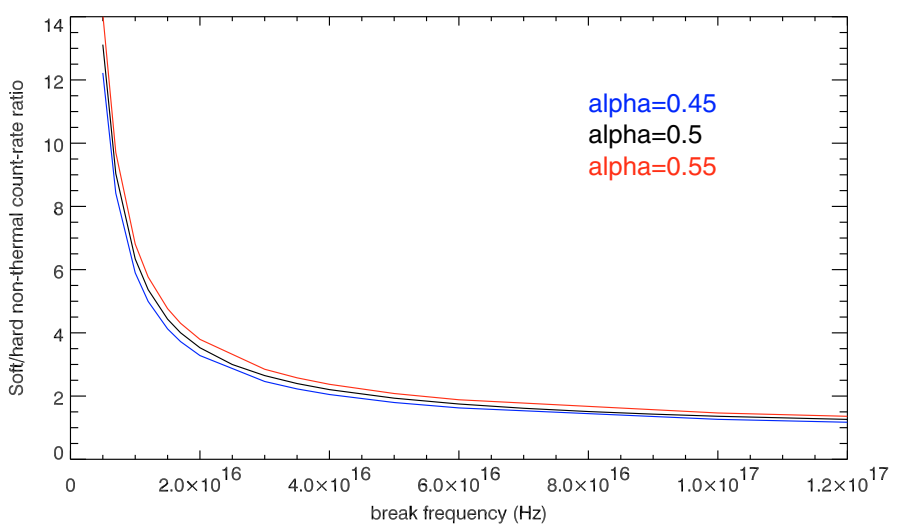

Fig. A.2. Ratio of the EPIC MOS count-rate in the $0.5-0.8 \mathrm{keV}$ and 2-4.5 keV bands as a function of the break frequency, obtained by assuming an SRCUT model with spectral index $\alpha=0.45$ (blue curve), $\alpha=0.5$ (black curve), and $\alpha=0.55$ (red curve).

region is correct. In conclusion, we can say that our assumption holds where it is more necessary.

\section{A.2. Step B}

From the $2-4.5 \mathrm{keV}$ image, we can produce NONT $H$ by considering a given spectral shape of the synchrotron emission (i.e., a given value of $\alpha$ and a given value of the roll-off frequency $v_{\text {break }}$ ) in each pixel. Since we found that in the entire rim the photon index is fairly uniform (see Fig. 3), we can assume that in each point of the image in the hard band the spectrum has a photon index $\alpha=0.5$. As for $v_{\text {break }}$, we found in Sect. 3.1 that it depends on the azimuthal angle $\theta$ at the rim. We then interpolate the values of $v_{\text {break }}$ shown in Fig. 3 by using a Fourier series (up to the 6 th order) and we assume that the azimuthal trend of $v_{\text {break }}(\theta)$ at the rim is given by this interpolating function at angular distances from the center $R>R_{\text {int }}(\theta)$, where $R_{\text {int }}(\theta)=11.7^{\prime}$ for regions 13-17 and $R_{\text {int }}(\theta)=13.5^{\prime}$ elsewhere. As for the radial profile of $v_{\text {break }}(R)$ for $R<R_{\text {int }}(\theta)$, we follow Rothenflug et al. (2004) and assume that: i) in the center $\left(R<7^{\prime}\right)$, the cut-off frequency is uniform and its value is equal to the minimum $v_{\text {break }}$ found in the rim and ii) for $7^{\prime}<R<R_{\text {int }}(\theta)$, the cut-off frequency increases exponentially with $R$, going from its minimum value (at the center) to the corresponding value at the rim. In this way, we produce the map of $v_{\text {break }}$ shown in Fig. A.1. Since we know the spectral shape of the synchrotron emission in each pixel of the $2-4.5 \mathrm{keV}$ image, we can produce the image of the non-thermal emission in the $0.5-0.8 \mathrm{keV}$ band. This image is shown in the lower panel of Fig. A.1. We investigate how the uncertainties in the best-fit parameters influence NONTH. Figure A. 2 shows the ratio, $\rho$, of the non-thermal EPIC MOS count-rate in the $0.5-0.8 \mathrm{keV}$ and $2-4.5 \mathrm{keV}$ bands as a function of $v_{\text {break }}$, obtained with $\alpha=0.5$ (black curve), $\alpha=0.45$ (blue curve), and $\alpha=0.55$ (red curve). This ratio provides the factor that allows us to produce, for each pixel, NONTH from the $2-4.5 \mathrm{keV}$ map. Since in Fig. A.2 the red curve, the blue curve, and the black curve are very similar, we can conclude that the uncertainties in the determination of $\alpha$ do not significantly alter our results. As for the effects of the uncertainties in $v_{\text {break, }}$, we focus only on the non-thermal limbs, since in thermal limbs, as explained in Sect. A.1, the non-thermal count-rate in the $0.5-0.8 \mathrm{keV}$ band is negligible. Figure A. 2 shows that in the non-thermal limbs (where $v_{\text {break }} \gtrsim 4 \times 10^{16} \mathrm{~Hz}$ and where the errors in $v_{\text {break }}$ are quite small, see Fig. 3), $\rho$ does not depend 
significantly on $v_{\text {break }}$. Therefore, in these regions, the effects of the uncertainties in $v_{\text {break }}$ are also negligible.

\section{A.3. Step $C$}

Once NONTH has been produced, we simply subtract it from the total image in the oxygen band, thus obtaining the pure thermal image in the $0.5-0.8 \mathrm{keV}$ band shown in Fig. 4.

\section{References}

Acero, F., Ballet, J., \& Decourchelle, A. 2007, A\&A, 475, 883 Allen, G. E., Houck, J. C., \& Sturner, S. J. 2008, ApJ, 683, 773

Arnaud, M., Neumann, D. M., Aghanim, N., et al. 2001, A\&A, 365, L80 Bamba, A., Yamaguchi, H., Koyama, K., et al. 2008, Adv. Space Res., 41, 411 Berezhko, E. G., \& Ellison, D. C. 1999, ApJ, 526, 385

Berezhko, E. G., \& Völk, H. J. 2007, ApJ, 661, L175

Blandford, R., \& Eichler, D. 1987, Phys. Rep., 154, 1

Blasi, P. 2002, Astrop. Phys., 16, 429

Borkowski, K. J., Lyerly, W. J., \& Reynolds, S. P. 2001, ApJ, 548, 820

Carter, J. A., \& Read, A. M. 2007, A\&A, 464, 1155
Cassam-Chenaï, G., Hughes, J. P., Reynoso, E. M., Badenes, C., \& Moffett, D. 2008, ApJ, 680, 1180

Decourchelle, A., Ellison, D. C., \& Ballet, J. 2000, ApJ, 543, L57

Dubner, G. M., Giacani, E. B., Goss, W. M., Green, A. J., \& Nyman, L.-Å. 2002, A\&A, 387, 1047

Dyer, K. K., Reynolds, S. P., \& Borkowski, K. J. 2004, ApJ, 600, 752

Ellison, D. C., Decourchelle, A., \& Ballet, J. 2004, A\&A, 413, 189

Fryxell, B., Olson, K., Ricker, P., et al. 2000, ApJS, 131, 273

Kane, J., Drake, R. P., \& Remington, B. A. 1999, ApJ, 511, 335

Koyama, K., Petre, R., Gotthelf, E. V., et al. 1995, Nature, 378, 255

Orlando, S., Bocchino, F., Reale, F., Peres, G., \& Petruk, O. 2007, A\&A, 470, 927

Petruk, O., Dubner, G., Castelletti, G., et al. 2008, ArXiv e-prints

Read, A. M., \& Ponman, T. J. 2003, A\&A, 409, 395

Reynolds, S. P., \& Keohane, J. W. 1999, ApJ, 525, 368

Rothenflug, R., Ballet, J., Dubner, G., et al. 2004, A\&A, 425, 121

Snowden, S., \& Kuntz, K. 2007, XMM-Newton ESAS manual, 2, ftp://xmm. esac. esa.int/pub/xmm-esas/xmm-esa s.pdf

Strüder, L., Briel, U., Dennerl, K., et al. 2001, A\&A, 365, L18

Turner, M. J. L., Abbey, A., Arnaud, M., et al. 2001, A\&A, 365, L27

Vink, J., Laming, J. M., Gu, M. F., Rasmussen, A., \& Kaastra, J. S. 2003, ApJ, 587, L31

Winkler, P. F., Gupta, G., \& Long, K. S. 2003, ApJ, 585, 324

Yamaguchi, H., Koyama, K., Katsuda, S., et al. 2008, PASJ, 60, 141 